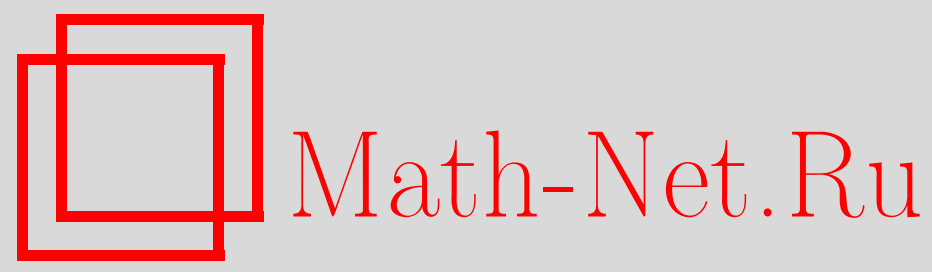

А. Г. Костюченко, К. А. Мирзоев, Трехчленные рекуррентные соотношения с матричными коэффициентами. Вполне неопределенный случай, Матем. заметки, 1998, том 63, выпуск 5, 709-716

DOI: https://doi.org/10.4213/mzm1337

Использование Общероссийского математического портала Math-Net.Ru подразумевает, что вы прочитали и согласны с пользовательским соглашением http://www.mathnet.ru/rus/agreement

Параметры загрузки:

IP: 3.85 .183 .62

26 апреля 2023 г., 18:12:37

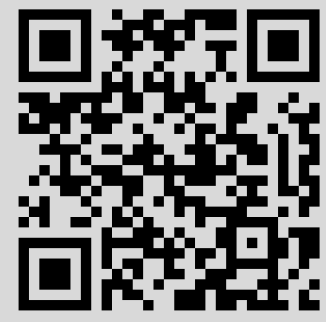




\section{ТРЕХЧЛЕННЫЕ РЕКУРРЕНТНЫЕ СООТНОШЕНИЯ \\ С МАТРИЧНЫМИ КОЭФФИЦИЕНТАМИ. ВПОЛНЕ НЕОПРЕДЕЛЕННЫЙ СЛУЧАЙ}

\section{А. Г. Костюченко, К. А. Мирзоев}

В пространстве последовательностей векторов $\ell_{p}^{2}$ рассматривается симметрический оператор $L$, порожденный выражением $(l u)_{j}:=B_{j} u_{j+1}+A_{j} u_{j}+B_{j-1}^{*} u_{j-1}$, где $u_{-1}=0, u_{0}, u_{1}, \ldots \in \mathbb{C}^{p}, A_{j}, B_{j}$-матрицы порядка $p$ с элементами из $\mathbb{C}$, причем $A_{j}^{*}=A_{j}, B_{j}^{-1}(j=0,1, \ldots)$ существуют. Приводится необходимое и достаточное условие, для того чтобы дефектные числа оператора $L$ были максимальными, т.е. для выражения $l$ имел место вполне неопределенньй случай. Получены также признаки не вполне неопределенности и вполне неопределенности для выражения $l$ в терминах коэффициентов $A_{j}, B_{j}$.

Библиограффия: 5 названий.

1. Введение. Пусть $\mathbb{C}^{p}(p \geqslant 1)$ - евклидово $p$-мерное пространство вектор-столбцов со стандартным скалярным произведением $y^{*} x=\sum_{j=1}^{p} x_{j} \bar{y}_{j}$, где $x_{j}, y_{j}(j=1,2, \ldots, p)$ координаты векторов $x$ и $y$ соответственно, а звездочка означает комплексно сопряженную матрицу. Пусть далее $A_{j}, B_{j}(j=0,1, \ldots)$ - матрицы порядка $p$, элементы которых являются комплексньми числами, причем $A_{j}$ - самосопряженная матрища, а $B_{j}^{-1}$ $(j=0,1, \ldots)$ существует. Рассмотрим разностное выражение второго порядка

$$
(l u)_{j}:=B_{j} u_{j+1}+A_{j} u_{j}+B_{j-1}^{*} u_{j-1}, \quad j=0,1, \ldots,
$$

где $u_{-1}:=0, u_{0}, u_{1}, \cdots \in \mathbb{C}^{p}$. Символом $\boldsymbol{l}$ обозначим операцию, действующую на последовательности квадратных матриц $U_{0}, U_{1}, \ldots$ размерности $p$ с элементами из $\mathbb{C}$ так же, как и $l$, т.е. по формуле (1). Справедлива следующая формула Грина:

$$
\sum_{j=k}^{n}\left((l u)_{j}^{*} v_{j}-u_{j}^{*}(l v)_{j}\right)=\left.[u v](s)\right|_{k-1} ^{n},
$$

где

$$
\left.[u v](s)\right|_{k-1} ^{n}:=[u v](n)-[u v](k-1)
$$

и

$$
[u v](s):=u_{s+1}^{*} B_{s}^{*} v_{s}-u_{s}^{*} B_{s} v_{s+1}, \quad s=0,1, \ldots
$$

Работа выполнена при финансовой поддержке Российского фонда фундаментальных исследований, грант № 96-01-00333. 
Аналогичная формула справедлива и в случае, когда векторы $u_{j}, v_{j}$ заменяются на матрицы $U_{j}, V_{j}$, а операция $l$ на операцию $l$.

Обозначим через $\ell_{p}^{2}$ гильбертово пространство бесконечных последовательностей векторов $u=\left(u_{0}, u_{1}, \ldots\right), u_{j} \in \mathbb{C}^{p}$, со скалярньм произведением $(u, v)=\sum_{j=0}^{+\infty} v_{j}^{*} u_{j}$, и пусть $L$ - минимальный замкнутый оператор, порожденный выражением (1) и граничным условием $u_{-1}=0$ в пространстве $\ell_{p}^{2}$ (см. [1, гл. VII, $\S 2$, п. 5, с. 509], [2]). Хорошо известно, что оператор $L$ симметричен, но вообще говоря, несамосопряжен, и дефектные числа $n_{+}$и $n_{-}$этого оператора удовлетворяют неравенствам $0 \leqslant n_{+}, n_{-} \leqslant p$. Говорят, что для выражения $l$ имеет место вполне неопределенный случай, если $n_{+}=n_{-}=p$. Из наших дальнейших рассуждений (см. теорему 1 и ее доказательство) легко извлечь, что для выражения $l$ имеет место вполне неопеделенный случай тогда и только тогда, когда все решения уравнения

$$
(l u)_{j}=z u_{j}, \quad j=0,1, \ldots, \quad u_{-1}=0,
$$

при $z=0$ принадлежат пространству $\ell_{p}^{2}$.

Развивая эту идею, в данной работе мы приводим критерий вполненеопределенности для выражения $l$ (теорема 2), которая является аналогом соответствующего утверждения для обыкновенных симметрических дифференциальньх уравнений (см. [3]). Далее в работе средствами этого критерия доказаны признаки не вполне неопределенности (теорема 3) и вполне неопределенности (теорема 4) для выражения $l$ уже в терминах коэффициентов этого выражения.

2. Предварительные сведения. Через $P_{j}(z)$ и $Q_{j}(z)(z \in \mathbb{C})$ обозначим решения матричного уравнения

$$
(\boldsymbol{l} U)_{j}=z U_{j}, \quad j=1,2, \ldots,
$$

удовлетворяющие начальным условиям $P_{0}(z):=I, P_{1}(z):=B_{0}^{-1}\left(z I-A_{0}\right)$ и $Q_{0}(z):=O$, $Q_{1}(z):=B_{0}^{-1}$ соответственно, где $O$ и $I$, как обычно, - нулевая и единичная матрица размерности $p$. Матриц-функции $P_{j}(z)$ и $Q_{j}(z)$ являются многочленами $j$-го и $(j-1)$-го порядка от комплексного переменного $z$ с матричными коэффициентами и называются матричными многочленами первого и второго рода соответственно. Эти многочлены удовлетворяют следующим соотношениям:

$$
\begin{array}{cc}
Q_{j+1}^{*}(z) B_{j}^{*} P_{j}(z)-Q_{j}^{*}(z) B_{j} P_{j+1}(z)=I, & j=0,1, \ldots, \\
Q_{j+1}^{*}(z) B_{j}^{*} Q_{j}(z)-Q_{j}^{*}(z) B_{j} Q_{j+1}(z)=O, & \\
P_{j+1}^{*}(z) B_{j}^{*} P_{j}(z)-P_{j}^{*}(z) B_{j} P_{j+1}(z)=O, & j=0,1, \ldots,
\end{array}
$$

где всюду $U^{*}(z)=(U(\bar{z}))^{*}$.

Формулы (4), (5) можно извлечь, очевидно, из формулы Грина подходящим выбором матриц $U_{j}$ и $V_{j}$. Разумеется, эти формулы, как и саму формулу Грина, можно доказать, манипулируя уравнением (3) и определением выражения $(\boldsymbol{l} U)_{j}$ соответственно.

Пусть $F_{j}(j=1,2, \ldots)$ - произвольная последовательность матриц порядка $p$. Считая матрицы $P_{j}(z)$ и $Q_{j}(z)(z \in \mathbb{C})$ известными, методом вариации постоянных решим уравнение

$$
(l U)_{j}-z U_{j}=F_{j}, \quad j=1,2, \ldots
$$

Положив

$$
U_{j}(z)=P_{j}(z) C_{j}^{(1)}+Q_{j}(z) C_{j}^{(2)}, \quad j=1,2, \ldots
$$


матрицы $C_{j}^{(i)}(i=1,2)$ выберем так, чтобы

$$
P_{j}(z) \Delta C_{j+1}^{(1)}+Q_{j}(z) \Delta C_{j+1}^{(2)}=O, \quad B_{j}\left(P_{j+1}(z) \Delta C_{j+1}^{(1)}+Q_{j+1}(z) \Delta C_{j+1}^{(2)}\right)=F_{j},
$$

где $\Delta C_{j}^{(i)}=C_{j}^{(i)}-C_{j-1}^{(i)}(i=1,2)$. Пусть, кроме того, $C_{1}^{(1)}=C_{0}^{(1)}-$ произвольная постоянная матрица. Непосредственно проверяется, что если матрицы $C_{j}^{(i)}(i=1,2)$ выбирать таким образом, то функция $U_{j}(z)$, определяемая равенством $(7)$, является решением уравнения (6).

Покажем, что система (8) всегда имеет единственное решение. Действительно, умножая первое уравнение этой системы слева на $Q_{j+1}^{*}(z) B_{j}^{*}$, а второе уравнение также слева на $-Q_{j}^{*}(z)$ и сложив полученные равенства, имеем

$$
\begin{aligned}
& \left(Q_{j+1}^{*}(z) B_{j}^{*} P_{j}(z)-Q_{j}^{*}(z) B_{j} P_{j+1}(z)\right) \Delta C_{j+1}^{(1)} \\
& \quad+\left(Q_{j+1}^{*}(z) B_{j}^{*} Q_{j}(z)-Q_{j}^{*}(z) B_{j} Q_{j+1}(z)\right) \Delta C_{j+1}^{(2)}=-Q_{j}^{*}(z) F_{j}
\end{aligned}
$$

Применяя теперь формулы (4), находим, что

$$
\Delta C_{j+1}^{(1)}=-Q_{j}^{*}(z) F_{j}, \quad j=1,2, \ldots
$$

Аналогичными рассуждениями можно показать, что

$$
\Delta C_{j+1}^{(2)}=P_{j}^{*}(z) F_{j}, \quad j=1,2, \ldots
$$

Из равенств (9) и (10) следует справедливость нашего утверждения.

В частности, если в равенствах $(9)$ и $(10)$ взять $F_{j}=I(j=1,2, \ldots)$ и учесть их в $(8)$, находим, что справедливы тождества

$$
\begin{gathered}
Q_{j}(z) P_{j}^{*}(z)-P_{j}(z) Q_{j}^{*}(z)=O, \quad j=0,1, \ldots, \\
Q_{j+1}(z) P_{j}^{*}(z)-P_{j+1}(z) Q_{j}^{*}(z)=B_{j}^{-1}, \quad j=0,1, \ldots .
\end{gathered}
$$

Тождество (12) является аналогом формулы Лиувилля-Остроградского.

Суммируя по $i=k, k+1, \ldots, j-1$, из равенств (9) и (10) находим, что

$$
C_{j}^{(1)}=C_{k}^{(1)}-\sum_{i=k}^{j-1} Q_{i}^{*}(z) F_{i}, \quad C_{j}^{(2)}=C_{k}^{(2)}+\sum_{i=k}^{j-1} P_{i}^{*}(z) F_{i}
$$

Поэтому в силу (7) имеем

$$
\begin{gathered}
U_{j}(z)=P_{j}(z) C_{k}^{(1)}+Q_{j}(z) C_{k}^{(2)}+\sum_{i=k}^{j-1}\left(Q_{j}(z) P_{i}^{*}(z)-P_{j}(z) Q_{i}^{*}\right) F_{i} \\
k=0,1, \ldots, \quad j=k+1, k+2, \ldots
\end{gathered}
$$

3. Критерий вполне неопределенности. Теперь докажем, что справедлива

ТЕорема 1. Если каждое решение векторного уравнения (2) для некоторого вещественного $z_{0}$ принадлежит пространству $\ell_{p}^{2}$, то для произвольного комплексного z кажсдое решение уравнения (2) принадлехсит пространству $\ell_{p}^{2}$. 
ДокАЗАТЕЛЬство. Пусть все решения уравнения (2) при некотором вещественном $z=z_{0}$ принадлежат пространству $\ell_{p}^{2}$. Тогда легко видеть, что $M_{k} \rightarrow 0$ при $k \rightarrow+\infty$, где

$$
M_{k}:=\max \left\{\left(\sum_{j=k}^{+\infty}\left\|P_{j}\left(z_{0}\right)\right\|^{2}\right)^{1 / 2},\left(\sum_{j=k}^{+\infty}\left\|Q_{j}\left(z_{0}\right)\right\|^{2}\right)^{1 / 2}\right\}
$$

и символом $\|$ • \| обозначена произвольная матричная норма. В частности, в качестве $\|$ · $\|$ далее используем самосопряженную матричную норму, т.е. такую норму, для которой $\left\|U^{*}\right\|=\|U\|$, где $U-$ произвольная матрица.

Матричное уравнение (3) запишем в виде

$$
(\boldsymbol{l} U)_{j}-z_{0} U_{j}=\left(z-z_{0}\right) U_{j} m, \quad j=0,1, \ldots,
$$

и применим формулу $(13)$, положив $F_{j}=\left(z-z_{0}\right) U_{j}(z)(j=1,2, \ldots)$. Тогда

$$
\begin{gathered}
U_{j}(z)=P_{j}\left(z_{0}\right) C_{k}^{(1)}+Q_{j}\left(z_{0}\right) C_{k}^{(2)}+\left(z-z_{0}\right) \sum_{i=k}^{j}\left(Q_{j}\left(z_{0}\right) P_{i}^{*}\left(z_{0}\right)-P_{j}\left(z_{0}\right) Q_{i}^{*}\left(z_{0}\right)\right) U_{i}(z), \\
j=k, k+1, \ldots
\end{gathered}
$$

С другой стороны, $\left\|P_{j}^{*}\left(z_{0}\right)\right\|=\left\|P_{j}\left(z_{0}\right)\right\|$ и $\left\|Q_{j}^{*}\left(z_{0}\right)\right\|=\left\|Q_{j}\left(z_{0}\right)\right\|$, так как число $z_{0}$ вещественное. Поэтому, применяя неравенство Коши-Буняковскогои свойства матричной нормы, находим, что

$$
\begin{aligned}
& \left\|\sum_{i=k}^{j}\left(Q_{j}\left(z_{0}\right) P_{i}^{*}\left(z_{0}\right)-P_{j}\left(z_{0}\right) Q_{i}^{*}\left(z_{0}\right)\right) U_{i}(z)\right\| \\
& \quad \leqslant\left\|Q_{j}\left(z_{0}\right)\right\| \sum_{i=k}^{j}\left\|P_{i}\left(z_{0}\right)\right\| \cdot\left\|U_{i}(z)\right\|+\left\|P_{j}\left(z_{0}\right)\right\| \sum_{i=k}^{j}\left\|Q_{i}\left(z_{0}\right)\right\| \cdot\left\|U_{i}(z)\right\| \\
& \quad \leqslant M_{k} N_{k, j}(z)\left(\left\|Q_{j}\left(z_{0}\right)\right\|+\left\|P_{j}\left(z_{0}\right)\right\|\right),
\end{aligned}
$$

где

$$
N_{k, j}(z):=\left(\sum_{i=k}^{j}\left\|U_{i}(z)\right\|^{2}\right)^{1 / 2} .
$$

Таким образом, из (14) находим, что

$$
\left\|U_{j}(z)\right\| \leqslant\left(C_{k}+\left|z-z_{0}\right| M_{k} N_{k, j}(z)\right)\left(\left\|P_{j}\left(z_{0}\right)\right\|+\left\|Q_{j}\left(z_{0}\right)\right\|\right),
$$

где $C_{k}=\max \left\{\left\|C_{k}^{(1)}\right\|,\left\|C_{k}^{(2)}\right\|\right\}$. Заменим в этом неравенстве переменную $j$ на $i$ и возведем обе части в квадрат. Суммируя затем почленно полученные неравенства по $i=k$, $k+1, \ldots, j$, извлекая квадратньй корень из обеих частей и применяя неравенство Минковского, находим, что

$$
N_{k, j}(z) \leqslant 2 C_{k} M_{k}+2\left|z-z_{0}\right| M_{k}^{2} N_{k, j}(z) .
$$

Если $k$ настолько велико, что $\left|z-z_{0}\right| M_{k}^{2} \leqslant 1 / 4$, то $N_{k, j}(z) \leqslant 4 C_{k} M_{k}$. Так как правая часть этого неравенства не зависит от $j$, выражение $N_{k, j}(z)$ имеет предел при $j \rightarrow+\infty$. Поэтому любое решение уравнения (2) принадлежит пространству $\ell_{p}^{2}$. Теорема 1 доказана. 
ЗАмечАние 1. Таким образом, если все решения уравнения (2) при некотором вещественном $z_{0}$ принадлежат пространству $\ell_{p}^{2}$, то для выражения $l$ имеет место вполне неопределенньй случай. Из равенства (14) можно извлечь, что справедливо и обратное утверждение, более того, в этом случае все решения уравнения (2) при любом $z \in \mathbb{C}$ принадлежат пространству $\ell_{p}^{2}$ (см. также [1, гл. VII, $§ 2$, п. 11, с. 589]).

По аналогии с обькновенными дифференциальными уравнениями в дальнейшем последовательность

$$
Q_{i} P_{j}^{*}-P_{i} Q_{j}^{*}=: K_{i j}, \quad i, j=0,1, \ldots,
$$

где $P_{i}:=P_{i}(0)$ и $Q_{i}:=Q_{i}(0)$, будем называть последовательностью Коши уравнения (6) (при $z=0$ ). Отметим, что эта последовательность при фиксированном $j$ удовлетворяет матричному уравнению (3) при $z=0$, начальным условиям

$$
K_{j j}=0, \quad K_{j+1, j}=B_{j}^{-1}, \quad j=0,1, \ldots
$$

(см. равенства (11) и (12) при $z=0$ ), и соотношению

$$
K_{i j}^{*}=-K_{j i}, \quad i, j=0,1, \ldots
$$

\section{Справедлива следующая}

ТЕОрема 2. Для того чтобы для выражения $l$ имел место вполне неопределенный случай, необходимо и достаточно, чтобы для любой последовательности отрезков натуральных чисел $\left[n_{k}, m_{k}\right]$ таких, что $m_{k} \leqslant n_{k+1}<m_{k+1}(k=1,2, \ldots)$, выполнялось условие

$$
\sum_{k=1}^{+\infty}\left(\sum_{i=n_{k}}^{m_{k}} \sum_{j=n_{k}}^{i}\left\|K_{i j}\right\|^{2}\right)^{1 / 2}<+\infty
$$

ДокАЗАТЕЛЬСтво. Необходимость. В силу неравенства Коши-Буняковского находим, что

$$
\left\|K_{i j}\right\|^{2} \leqslant\left(\left\|Q_{i}\right\| \cdot\left\|P_{j}\right\|+\left\|P_{i}\right\| \cdot\left\|Q_{j}\right\|\right)^{2} \leqslant\left(\left\|P_{i}\right\|^{2}+\left\|Q_{i}\right\|^{2}\right)\left(\left\|P_{j}\right\|^{2}+\left\|Q_{j}\right\|^{2}\right) .
$$

Следовательно,

$$
\sum_{i=n_{k}}^{m_{k}} \sum_{j=n_{k}}^{i}\left\|K_{i j}\right\|^{2} \leqslant \sum_{i=n_{k}}^{m_{k}}\left(\left\|P_{i}\right\|^{2}+\left\|Q_{i}\right\|^{2}\right) \sum_{j=n_{k}}^{i}\left(\left\|P_{j}\right\|^{2}+\left\|Q_{j}\right\|^{2}\right) \leqslant\left(\sum_{i=n_{k}}^{m_{k}}\left\|P_{i}\right\|^{2}+\left\|Q_{i}\right\|^{2}\right)^{2} .
$$

Извлекая далее квадратный корень из обеих частей полученного неравенства и суммируя их по $k$, находим, что

$$
\sum_{k=1}^{+\infty}\left(\sum_{i=n_{k}}^{m_{k}} \sum_{j=n_{k}}^{i}\left\|K_{i j}\right\|^{2}\right)^{1 / 2} \leqslant \sum_{k=1}^{+\infty}\left(\sum_{i=n_{k}}^{m_{k}}\left\|P_{i}\right\|^{2}+\left\|Q_{i}\right\|^{2}\right) \leqslant 2 \sum_{k=1}^{+\infty}\left(\left\|P_{j}\right\|^{2}+\left\|Q_{j}\right\|^{2}\right)<+\infty .
$$

Таким образом, если все решения уравнения (2) при $z=0$ принадлежат пространству $\ell_{p}^{2}$, то условия (15) теоремы 2 вьполняются. 
Достаточность. Сначала покажем, что если не все решения уравнения (2) при $z=0$ принадлежат пространству $\ell_{p}^{2}$, то для любого $l \geqslant 1$

$$
\sum_{i=l}^{+\infty} \sum_{j=l}^{+\infty}\left\|K_{i j}\right\|^{2}=+\infty
$$

Пусть при некотором $l=l_{0}$ это не так. Тогда в силу (16) находим, что

$$
\sum_{i=l_{0}}^{+\infty} \sum_{j=l_{0}}^{+\infty}\left\|K_{i j}\right\|^{2}=\sum_{i=l_{0}}^{+\infty} \sum_{j=l_{0}}^{i}\left\|K_{i j}\right\|^{2}+\sum_{i=l_{0}}^{+\infty} \sum_{j=i}^{+\infty}\left\|K_{i j}\right\|^{2}=2 \sum_{i=l_{0}}^{+\infty} \sum_{j=l_{0}}^{i}\left\|K_{i j}\right\|^{2}<+\infty .
$$

Из этого, в частности, следует, что

$$
\sum_{i=1}^{+\infty}\left\|K_{i, l_{0}}\right\|^{2}<+\infty, \quad \sum_{i=1}^{+\infty}\left\|K_{i, l_{0}+1}\right\|^{2}<+\infty .
$$

С другой стороны, применяя тождества (4), (5), легко установить, что

$P_{i}=K_{i, l_{0}} B_{l_{0}} P_{l_{0}+1}-K_{i, l_{0}+1} B_{l_{0}}^{*} P_{l_{0}}, Q_{i}=K_{i, l_{0}} B_{l_{0}} Q_{l_{0}+1}-K_{i, l_{0}+1} B_{l_{0}}^{*} Q_{l_{0}}, \quad i=1,2, \ldots$

Исходя из этих тождеств, стандартными рассуждениями можно показать, что

$$
\sum_{i=1}^{+\infty}\left(\left\|P_{i}\right\|^{2}+\left\|Q_{i}\right\|^{2}\right) \leqslant C \sum_{i=1}^{+\infty}\left(\left\|K_{i, l_{0}}\right\|^{2}+\left\|K_{i, l_{0}+1}\right\|^{2}\right),
$$

где $C$ - некоторая положительная постоянная. Это неравенство противоречит предположению о том, что не все решения уравнения (2) принадлежат пространству $\ell_{p}^{2}$. Теперь предположим, что для любой последовательности отрезков натуральных чисел $\left[n_{k}, m_{k}\right]$ условие (17) выполнено, но не все решения уравнения (2) при $z=0$ принадлежат пространству $\ell_{p}^{2}$. Используя соотношения (18), легко заметить, что тогда существует, например, последовательность непересекающихся отрезков натуральных чисел $\left[n_{k}, m_{k}\right]$ $(k=1,2, \ldots)$ таких, что

$$
\sum_{i=n_{k}}^{m_{k}} \sum_{j=n_{k}}^{i}\left\|K_{i j}\right\|^{2} \geqslant 1
$$

Это противоречит нашему предположению. Теорема 2 доказана.

Теорема 2 является аналогом соответствующего утверждения (см. [3]) для обькновенных симметрических дифференциальных уравнений.

4. Признаки вполне неопределенности. Справедливо следующее предложение.

ТЕОРемА 3. Пусть выполняется какое-либо из следующих условий:

$$
\begin{array}{ll}
\text { а) } & \sum_{k=1}^{+\infty}\left\|B_{k}^{-1}\right\|=+\infty, \\
\text { б) } & \sum_{k=1}^{+\infty}\left\|B_{k+1}^{-1} A_{k+1} B_{k}^{-1}\right\|=+\infty, \\
\text { в) } & \sum_{k=1}^{+\infty}\left\|B_{k+2}^{-1}\left(A_{k+2} B_{k+1}^{-1} A_{k+1}-B_{k+1}^{*}\right) B_{k}^{-1}\right\|=+\infty .
\end{array}
$$

Тогда для выражсения $l$ не имеет места вполне неопределенный случай. 
ДокАЗАтЕЛЬСтво. Пусть $\left[n_{k}^{(s)}, m_{k}^{(s)}\right](k=1,2, \ldots, s=1,2, \ldots, N)$ - конечное число непересекаюшихся при каждом $s$ последовательностей отрезков натуральных чисел таких, что каждое из слагаемых вида $\left\|K_{n+i, i}\right\|^{2}$ встречается хотя бы в одной суммевида

$$
\sum_{i=n_{k}^{(s)}}^{m_{k}^{(s)}} \sum_{j=n_{k}^{(s)}}^{i}\left\|K_{i j}\right\|^{2}, \quad k=1,2, \ldots, \quad s=1,2, \ldots, N
$$

Очевидно, такие последовательности отрезков можно построить при любом фиксированном $i$. Таким образом, в силу неравенства

$$
\sum_{s=1}^{N} \sum_{k=1}^{+\infty}\left(\sum_{i=n_{k}^{(s)}}^{m_{k}^{(s)}} \sum_{j=n_{k}^{(s)}}^{i}\left\|K_{i j}\right\|^{2}\right)^{1 / 2} \geqslant \sum_{n=1}^{+\infty}\left\|K_{n+i, i}\right\|
$$

каждьй раз, когда ряд в правой части последнего неравенства расходится, расходится и один из рядов

$$
\sum_{k=1}^{+\infty}\left(\sum_{i=n_{k}^{(s)}}^{m_{k}^{(s)}} \sum_{j=n_{k}^{(s)}}^{i}\left\|K_{i j}\right\|^{2}\right)^{1 / 2}, \quad s=1,2, \ldots, N .
$$

Применяя теперь теорему 2 , находим, что если указанный ряд расходится при некотором фиксированном $i$, то уравнение (2) при $z=0$ имеет решение, не принадлежашее пространству $\ell_{p}^{2}$. Остается заметить, что

$$
\begin{aligned}
& K_{n+1, n}=B_{n}^{-1}, \quad K_{n+2, n}=-B_{n+1}^{-1} A_{n+1} B_{n}^{-1} \\
& K_{n+3, n}=B_{n+2}^{-1}\left(A_{n+2} B_{n+1}^{-1} A_{n+1}-B_{n+1}^{*}\right) B_{n}^{-1}
\end{aligned}
$$

в силу уравнения (3) при $z=0$. Теорема 3 доказана.

Отметим, что в случае $p=1$ условие а) теоремы 3 совпадает с известным признаком Карлемана (см. например, [1, гл. VII, §1, теорема 1.3, с. 509]), а условие б) этой теоремы - с признаком Денниса и Уолла (см. [4, гл. I, дополнения и задачи, 2, с. 37]).

Справедлива также

TeOpema 4. Пусть $\left\|A_{k}\right\| \leqslant C(k=0,1, \ldots) u$

$$
\sum_{j=0}^{+\infty} \sum_{s=0}^{+\infty}\left\|B_{j}^{*^{-1}} B_{j+1} B_{j+2}^{*^{-1}} \cdots B_{j+2 s-1} B_{j+2 s}^{*^{-1}}\right\|<+\infty .
$$

Тогда для выражсения $l$ имеет место вполне неопределенный случай.

ДоказАТЕЛьСтво. Если $\left\|A_{k}\right\| \leqslant C$, то оператор $y_{k} \rightarrow A_{k} y_{k}(k=0,1, \ldots)$, очевидно, является ограниченным самосопряженным оператором в пространстве $\ell_{p}^{2}$. Поэтому согласно известной теореме (см., например, [5, гл. IV, $\S 14$, п. 7 , теорема 6, c. 165]) дефектные числа оператора $L$ и оператора, порожденного выражением

$$
\left(l_{1} u\right)_{j}=B_{j} u_{j+1}+B_{j-1}^{*} u_{j-1}, \quad j=1,2, \ldots,
$$


совпадают. Далее следует применить теорему 2 к выражению $l_{1}$, учитьвая, что $K_{j+2 s, j}=0, K_{j+2 s+1, j}=(-1)^{s} B_{j+2 s}^{-1} B_{j+2 s-1}^{*} B_{j+2 s-2}^{-1} \cdots B_{j+1}^{*} B_{j}^{-1}, \quad s, j=0,1, \ldots$, так как последовательность Коши $K_{i j}$ удовлетворяет матричному уравнению

$$
B_{i} K_{i+1, j}+B_{i-1}^{*} K_{i-1, j}=O
$$

при фиксированном $j$ и начальным условиям (15). Теорема 4 доказана.

Теорема 4 является аналогом одной теоремы Березанского для случая $p=1$ (см., например, [1, гл. VII, §1, теорема 1.5, с. 513]).

Остановимся на одном следствии теоремы 4.

СледСтвиЕ. Пусть $\left\|A_{k}\right\| \leqslant C u\left\|B_{k+1}^{-1} B_{k}^{*}\right\| \leqslant q(k=0,1, \ldots)$, дде $C>0 u$ $0<q<1$ - постоянные. Тогда для выражсения $l$ имеет место вполне неопределенный случай.

ДокАЗАТЕЛЬСТво. Используя свойства самосопряженной матричной нормы, находим, что

$$
\begin{aligned}
\left\|B_{j}^{*^{-1}} B_{j+1} B_{j+2}^{*^{-1}} \cdots B_{j+2 s-1} B_{j+2 s}^{*^{-1}}\right\| & \leqslant\left\|B_{j}^{*^{-1}}\right\| \cdot\left\|B_{j+1} B_{j+2}^{*^{-1}}\right\| \cdots\left\|B_{j+2 s-1} B_{j+2 s}^{*^{-1}}\right\| \\
& \leqslant\left\|B_{j}^{-1}\right\| q^{s}, \quad i, s=0,1, \ldots
\end{aligned}
$$

и

$$
\left\|B_{j}^{-1}\right\|=\left\|B_{j}^{-1} B_{j-1}^{*} B_{j-1}^{*^{-1}}\right\| \leqslant q\left\|B_{j-1}^{-1}\right\| .
$$

Поэтому

$$
\sum_{j=0}^{+\infty} \sum_{s=0}^{+\infty}\left\|B_{j}^{-1}\right\| q^{s}=\frac{1}{1-q} \sum_{j=0}^{+\infty}\left\|B_{j}^{-1}\right\|<+\infty .
$$

Таким образом, все условия теоремы 4 вьполняются. Следствие доказано.

ЗАмЕчАниЕ 2. Все результаты данной работы без существенных изменений переносятся на случай, когда пространство $\mathbb{C}^{p}$ заменяется на сепарабельное гильбертово пространство $H$, а матрицы $A_{j}, B_{j}(j=0,1, \ldots)$ - на линейные ограниченные операторы в нем.

\section{СПИСОК ЦИТИРОВАННОЙ ЛИТЕРАТУРЫ}

[1] Березанский Ю. М. Разложение по собственньм функциям самосопряженных операторов. Киев: Наукова думка, 1965.

[2] Крейн М.Г. Бесконечные $J$ матрицы и матричная проблема моментов // Докл. АН СССР. 1949. T. 69 . № 3. C. $125-128$.

[3] Мирзоев К.А. Функция Коши и $L_{w}^{p}$ свойства решений квазидифференциальных уравнений // УМН. 1991. Т. 46. № 4(280). С. 161-162.

[4] Ахиезер Н. И. Классическая проблема моментов. М.: Физматлит, 1961.

[5] Наймарк М. А. Линейные дифференциальные операторы. М.: Наука, 1969.

Московский государственный университет им. М.В. Ломоносова 